SLAC-PUB- 14345

January 2011

\title{
LASER SAFETY: A LASER ALIGNMENT PRACTICAL TRAINING COURSE ${ }^{1}$
}

\author{
Michael Woods and Steve Edstrom, \\ SLAC National Accelerator Laboratory
}

\begin{abstract}
SLAC National Accelerator Laboratory has developed a Laser Alignment Practical Training Course as one of its core laser safety classes. The course is taught to small groups of up to three students and takes 1-3 hours to complete. This practical course is not a substitute for site-specific On-the-Job Training; it does, however, provide a good introduction in core laser safety practices that can be broadly applied. Alignment and diagnostic tasks are performed with low power lasers. Students learn safe alignment and diagnostic techniques and how to avoid common mistakes that might lead to an accident. The class is taught by laser supervisors, enabling them to assess the skill level of new laser personnel and determine the subsequent level of supervision needed. The course has six alignment tasks. For each task, discussion points are given for the instructor to review with the students. The optics setup includes different wavelength lasers, a beam expander, mirrors, irises, a
\end{abstract}

periscope, a beam-splitting polarizer and a diffraction grating. Diagnostic tools include viewing cards, an IR viewer and a ccd camera. Laser eyewear is available to block some laser wavelengths in the setup.

\section{Overview}

The alignment course is given by laser supervisors and is required prior to a laser operator performing alignment work with Class $3 \mathrm{~B}$ or Class 4 lasers. The course was developed by the LCLS Laser Department at SLAC [1]. It is one of three core laser safety classes required at SLAC for operators of high power Class 3B and Class 4 lasers. The other two courses are a webbased general laser safety course and a classroom course given by SLAC's Laser Safety Officer (LSO) on lessons learned from laser accidents. The alignment course and the lessons learned course were implemented following a laser accident at SLAC [2].

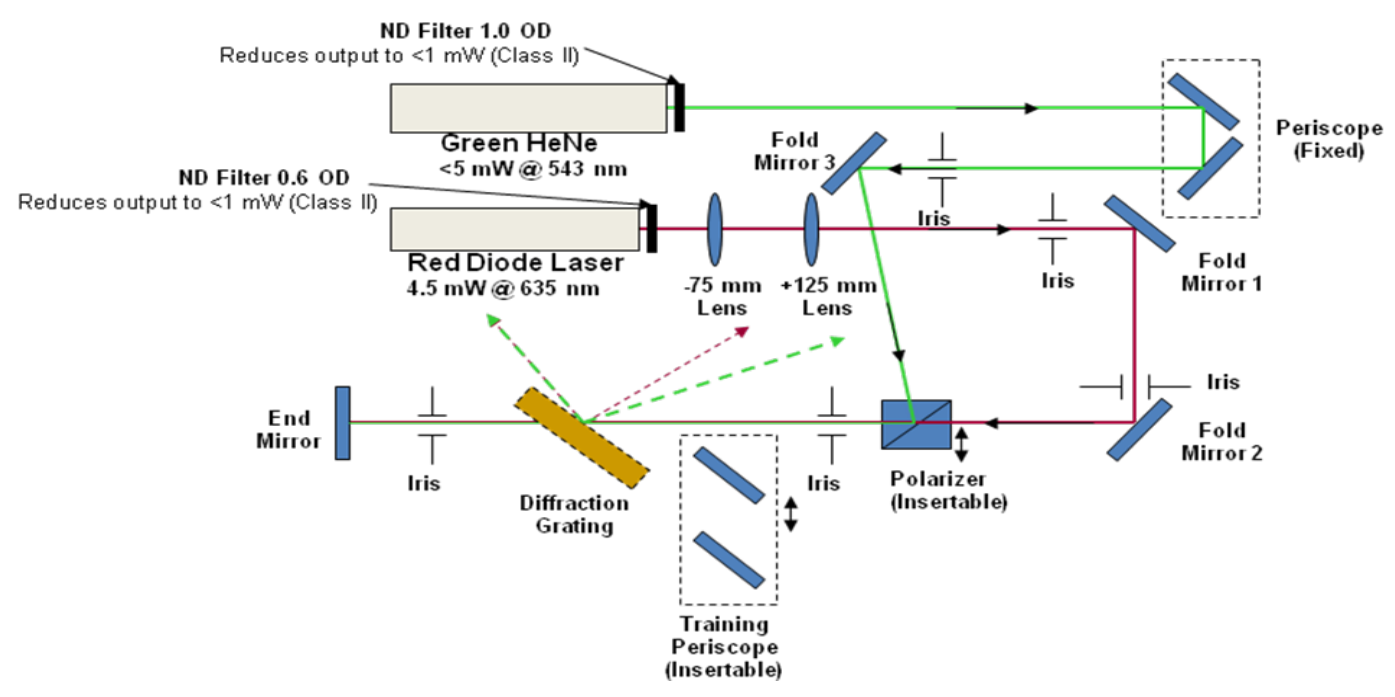

Figure 1: Optics configuration for laser alignment practical course

1 Contributed to the 2011 International Laser Safety Conference, San Jose, CA; http://www.laserinstitute.org/conferences/ilsc/conference 


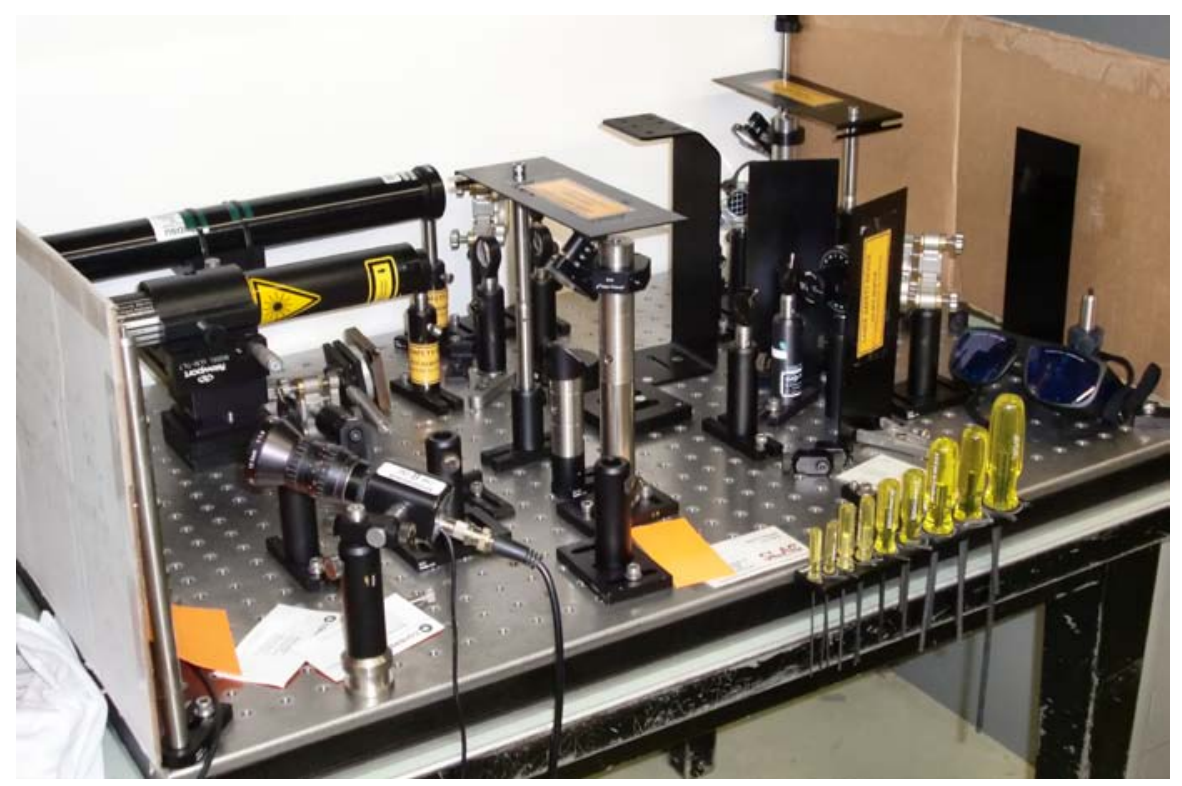

Figure 2: Photo of optics configuration for laser alignment practical course

The optics configuration used is shown in Figure 1 and a photo is given in Figure 2. There are six exercises:

i. Beam collimation and telescope alignment

ii. 2-pinhole alignment

iii. Periscope alignment

iv. Use of beam-splitting polarizers

v. Co-alignment of two laser beams

vi. Gratings and diffractive optics principals

Students must complete at least three exercises, which must include a periscope alignment task. The course takes 1-3 hours to complete depending on the number of students and their skill level. The maximum number of students is 3 . Each exercise has a list of tasks and an associated list of discussion points for the instructor to review with the students. Overall objective is to teach laser alignment best practices to laser operators and to be an interactive vehicle for supervisors to assess the skill level of new operators for their facility.

\section{Specific student objectives include:}

- demonstrating safe alignment practices (to be used later with high power Class $3 \mathrm{~B}$ and Class 4 lasers),

- learning techniques of assisted viewing (to use later with invisible laser beams while wearing laser eyewear protection),

- identifying appropriate controls to mitigate laser beam hazards, and

- recognizing how to avoid common mistakes that might lead to an accident.
Specific objectives for supervisors include:

- $\quad$ assessing skill level of new personnel, and

- determining subsequent level of supervision needed.

\section{Description of Alignment Exercises}

We now describe the tasks associated with each exercise and the discussion points the instructor will review with students. Each exercise has an associated schematic and list of tools which can be used; these tools include:

- fixed beam blocks and movable magnetic ones,

- IR and fluorescent cards,

- IR viewer,

- ccd camera and monitor, and

- laser eyewear protection for the red diode laser.

\subsection{Telescope Alignment}

The tasks given to the student are:

i. Block or disable green beam

ii. Remove lenses from the beam path

iii. Align beam through irises to a beam block and mark the beam spot

iv. Insert the first lens into the beam path and optimize position \& alignment

v. Insert the second lens into the beam path and optimize position \& alignment

vi. Check beam collimation 
The discussion points used are:

i. Does student remove rings, badges, and jewelry before starting alignment?

ii. How do you tell that the beam is collimated?

iii. What is the expected beam magnification and lens separation?

iv. How do you center lenses? Why?

v. How do you control back reflections?

vi. Which back reflections should you be particularly worried about and why?

vii. Does the student block the laser beam when inserting and removing optics elements?

\subsection{2-Pinhole Alignment}

The tasks given to the student are:

i. Block or disable the green beam

ii. Optimize alignment of the red beam through the two irises using the two folding mirrors after the telescope

iii. Optimize the end mirror alignment by observing the back-reflected beam position on the irises

iv. Verify all beams and reflected or leaked beams are contained

v. Try doing this with full protection eyewear on

The discussion points used are:

i. How to chase beam through the beam path

ii. How to use an IR card, IR viewer, or CCD camera to verify and set alignment

iii. How to properly hold an IR card, such that a reflected beam from it would go down rather than up. Note the reflected beams from the card and the potential hazard.

iv. Which back reflections should you be particularly worried about and why?

v. Are there potential leakage points? Where?

vi. Check for and block any stray beams

\subsection{Periscope Alignment}

The tasks given to the student are:

i. Block or disable both beams; and remove upper periscope mirror

ii. Insert training periscope

iii. Unblock red beam; verify its alignment entering the periscope; then optimize upward beam alignment.

iv. Install upper periscope mirror and optimize alignment toward final iris and beam block

v. Verify all beams are contained

vi. Block red beam and remove training periscope.

The discussion points used are:

i. Does the student block the laser before removing/inserting optical elements?

ii. How do you safely align the vertical reflecting mirror?

iii. How do you place the upper mirror?

iv. How do you contain the beam?

\subsection{Beam-splitting Polarizer Alignment}

The optics configuration for this task is shown in Figure 3. The tasks given to the student are:

i. Block or disable both beams.

ii. Instructor shall randomly orientate the polarizer in its mount before student installs polarizer in beam.

iii. Prior to installing polarizer, adjust escape window so reflected beam will be in horizontal plane.

iv. Install polarizer and associated beam block for the reflected beam.

v. Unblock the red beam.

vi. Verify all beams and reflected or leaked beams are contained

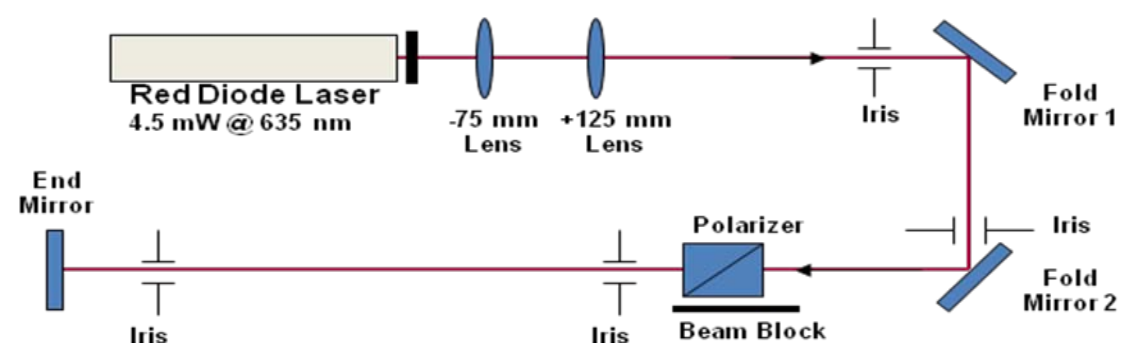

Figure 3: Optics configuration for beam-splitting polarizer task 
The discussion points used are:

i. Before polarizer is inserted into beam, discuss how polarizer is mounted and adjusted, and how the beam tube is used.

ii. Note ways that this mount might unintentionally produce a vertical output beam; discuss ways to avoid this.

iii. How to inspect for intentional and unintentional stray beams

\subsection{Co-aligning Two Beams}

The tasks given to the student are:

i. Block or disable green beam. Then verify red beam alignment thru last 2 irises.

ii. Block or disable the red beam. Remove green beam block and verify green beam alignment to polarizer.

iii. Optimize alignment of the green beam through the last two irises using only upper mirror in fixed periscope and Fold Mirror 3.

iv. Verify end mirror alignment by observing the back-reflected beam position on the irises

v. Verify all beams and reflected or leaked beams are contained

The discussion points used are:

i. Think about ways to do this with full protection goggles on.

ii. What are some other ways to combine beams on a sample, particularly beams of different wavelengths?

iii. Discuss ways to use CCDs and viewer cards.

iv. Discuss relative intensities of the 2 beams and apparent perception of their brightness. Discuss hazards of dimly visible beams outside normal 400-700nm visible range.

\subsection{Diffraction Grating Alignment}

The tasks given to the student are:

i. Block or disable green beam. Verify red beam alignment through last two irises

ii. Verify direction of rules on the grating if it is not marked

iii. Insert the mounted grating into its post holder and lock near normal incidence

iv. Optimize grating zero degree retro reflection alignment back through irises

v. Rotate to place diffracted beam back on iris.

vi. Optimize grating rule rotation to optimize diffracted beam vertically on the iris

vii. Check again zero degree retro reflection alignment back through irises viii. Position the grating angle to allow access to the zero order and diffracted beams

ix. Place beam blocks to contain all reflections

The discussion points used are:

i. Does student block beams before inserting/removing optics?

ii. How do you determine grating ruling orientation?

iii. How would you best control the multiple reflections?

iv. How do you safely handle a grating?

v. Note where such gratings exist in common laser systems.

\section{Future Upgrades to the Alignment Course}

Four upgrades are currently planned:

i. Add a 785nm diode laser, which will have a power of $3 \mathrm{~mW}$ (Class 3R). We will do tests to determine if operation at $0.6 \mathrm{~mW}$ is adequate for the diagnostics being used, which would allow Class 1 operation.

ii. Add a $405 \mathrm{~nm}$ diode laser with which will have a power of $5 \mathrm{~mW}$ (Class 3R). We will do tests to determine if operation at 1 $\mathrm{mW}$ is adequate for the diagnostics being used, which would allow Class 2 operation.

iii. Replace the 543nm HeNe laser with a 532nm diode laser, operating as a Class 2 laser with power less than $1 \mathrm{~mW}$.

iv. Add additional camera diagnostics to demonstrate different camera options.

The laser upgrades will make the same laser wavelengths available as for Ti:sapphire operation at $785 \mathrm{~nm}$ with a 532nm pump beam, and for operation with the $2^{\text {nd }}$ harmonic of the Ti:sapphire beam at $\sim 400$ $\mathrm{nm}$. This will allow students to try the same types of eyewear and diagnostics they will encounter with Ti:sapphire lasers, which is the most commonly used laser system at SLAC.

\section{Core Laser Safety Practices}

This practical training course provides a good introduction to laser alignment and diagnostic tasks that will be encountered in Class 3B and Class 4 laser operations. Students receive instruction in the core laser safety practices shown in Table 1 . A poster which lists these practices is displayed at the training course setup and at SLAC's laser labs. 
Table 1: Core Laser Safety Practices

\begin{tabular}{|c|c|}
\hline 1 & $\begin{array}{l}\text { Select proper eyewear; check condition each usage. } \\
\text { Ensure all personnel are wearing appropriate eyewear. }\end{array}$ \\
\hline 2 & Be knowledgeable of all safety controls and equipment safety features. \\
\hline 3 & Avoid bringing eyes near plane in which the laser propagates. \\
\hline 4 & Remove or cover jewellery, watches, etc. if the objects may be near the beam path. \\
\hline 5 & $\begin{array}{l}\text { Communicate. Alert others prior to turning on laser, opening shutters, or creating new } \\
\text { beam paths. }\end{array}$ \\
\hline 6 & Exclude unnecessary personnel during alignment. \\
\hline 7 & $\begin{array}{l}\text { Use indirect methods for viewing the beam such as phosphor cards, CCD cameras or } \\
\text { IR viewers. }\end{array}$ \\
\hline 8 & $\begin{array}{l}\text { Check for and block stray beams. When placing a new optical component in the beam, } \\
\text { locate and block all stray reflections before proceeding to next step. }\end{array}$ \\
\hline 9 & $\begin{array}{l}\text { Use beam blocks. Block the beam upstream until beam is needed; } \\
\text { place a block down beam of optic path being aligned. }\end{array}$ \\
\hline 10 & Keep primary and stray beams in horizontal plane below eye level when possible. \\
\hline 11 & $\begin{array}{l}\text { Special caution is needed when using periscopes, beam-splitting polarizers, and other } \\
\text { optics that may generate out-of-plane beams -- secure appropriate beam blocks to } \\
\text { contain possible stray beams. }\end{array}$ \\
\hline 12 & Use table enclosures, barriers and beam tubes when possible. \\
\hline 13 & Use irises to aid in alignment. \\
\hline 14 & Use minimum intensity needed, and use low power alignment lasers when possible. \\
\hline 15 & Secure all optics to table. Practice good housekeeping. \\
\hline
\end{tabular}

\section{Evaluations of the Alignment Course}

The course has received very positive evaluations from laser supervisors who give the course and from the students taking the course. The course is particularly beneficial for students who are new to laser work. It is also useful for experienced laser operators who may learn new techniques, identify bad habits, or improve their ability to teach others.

\section{Summary}

A new laser alignment practical training course has been developed at SLAC and is described in this paper. It is one of the core laser safety courses required before a laser operator begins alignment work with high power Class 3B or Class 4 lasers.

\section{Acknowledgements}

Work supported by the U.S. Department of Energy under contract number DE-AC02-76SF00515.

\section{References}

[1] The LCLS (Linac Coherent Light Source) is an Xray Free Electron Laser facility at SLAC. The LCLS Laser Department supports the operation of optical lasers used for LCLS accelerator operations and for LCLS experiments. See

http://www-

public.slac.stanford.edu/lcls/aboutlcls.aspx.

[2] M. Woods (2011) Lessons Learned from a Recent Laser Accident, SLAC-PUB-14346; Paper \# 201 contributed to this conference. 


\section{Meet the Authors}

Michael Woods, CLSO, is the Laser Safety Officer at the SLAC National Accelerator Laboratory. $\mathrm{He}$ is an Engineering Physicist, with a B.Sc. in Engineering Physics from Queen's University in Kingston, Ontario, Canada and a Ph.D. in High Energy Physics from the University of Chicago. He has spent 15 years as a researcher in experimental particle physics and accelerator physics utilizing high power laser systems. He became SLAC LSO in 2008.

Steve Edstrom is a laser engineer with the LCLS laser department, and is the laser supervisor for the central laser facility that provides Ti:sapphire laser systems for the LCLS Near Experimental Hall. Before joining SLAC in 2008 he worked at Coherent Inc. where he spent 6 years as their custom laser product line manager. Previous to that he worked for 13 years in various engineering, field support and engineering management roles for the laser industry (Positive Light, Continuum). 